\title{
PARABOLICITY, PROJECTIVE VOLUME AND FINITENESS OF TOTAL CURVATURE OF MINIMAL SURFACES
}

\author{
Atsushi Atsuji
}

\begin{abstract}
We show that finiteness of a projective volume implies finiteness of total curvature for stochastic complete minimal surfaces with finite number of ends and finite genus which may not be geodesically complete. The tools we use include simple stochastic calculus and Nevanlinna theoretic method.
\end{abstract}

Let $\mathbf{x}: M \rightarrow \mathbf{R}^{n}$ be a minimal surface. Q. Chen in [6] showed that if $M$ is a properly immersed minimal surface with finite number of ends and finite genus and $M$ has quadratic volume growth, then total curvature of $M$ is finite. Here $M$ is said to have quadratic volume growth if $\operatorname{vol}(\{|\mathbf{x}| \leq r\})=O\left(r^{2}\right)$. From a result by Jorge and Meeks [12] it follows that for proper immersed minimal surfaces with finite number of ends and finite genus, quadratic volume growth is equivalent to finiteness of its total curvature.

On the other hand, Tkachev [15] introduced projective volume $V_{p}(M)$ of properly immersed minimal submanifolds of dimension $m$ defined by

$$
V_{p}(M)=\lim _{r \rightarrow \infty} \frac{1}{\log r} \int_{\{|\mathbf{x}| \leq r\}} \frac{1}{|\mathbf{x}|^{m}} d v \quad(\leq \infty) .
$$

While this makes sense only for properly immersed minimal submanifolds, he also introduced

$$
Q_{p}(M, \mathbf{a})=\int_{M} \frac{\left|(\mathbf{x}-\mathbf{a})^{\perp}\right|^{2}}{|\mathbf{x}-\mathbf{a}|^{2+m}} d v \quad(\leq \infty),
$$

for $\mathbf{a} \in \mathbf{R}^{n}$ where $(\mathbf{x}-\mathbf{a})^{\perp}(x)$ is a projection of $\mathbf{x}-\mathbf{a}$ on the normal space to the surface $\mathbf{x}(M)$ at a point $\mathbf{x}(x)$. This is well-defined even for improper minimal submanifolds. He showed that for properly immersed minimal submanifold $M$ of dimension $m$ with $\mathbf{a} \notin \mathbf{x}(M)$,

2000 Mathematics Subject Classification. Primary 49Q05; Secondary $58 \mathrm{~J} 65$.

Key words and phrases. Minimal surface, Brownian motion, Nevanlinna theory.

Partly supported by the Grants-in-Aid for Scientific Research of the Japan Society for the Promotion of Science.

Received September 17, 2003; revised March 9, 2004. 


$$
V_{p}(M)=m Q_{p}(M, \mathbf{a}) .
$$

He also showed that finiteness of $Q_{p}(M, \mathbf{a})$ is independent of the choice of $\mathbf{a} \in \mathbf{R}^{n}$. Namely if $Q_{p}(M, \mathbf{a})<\infty$ for some $\mathbf{a} \in \mathbf{R}^{n}$, then $Q_{p}(M, \mathbf{a})<\infty$ for any $\mathbf{a} \in \mathbf{R}^{n}$. We are interested in the case when the immersion may be improper and $M$ may be geodesically incomplete. Our result is

THEOREM 1. Let $\mathbf{x}: M \rightarrow \mathbf{R}^{n}$ be a minimal surface with finite number of ends and finite genus. Suppose that $M$ is stochastically complete and $Q_{p}(M, \mathbf{a})<\infty$ for some $\mathbf{a} \in \mathbf{R}^{n}$ with $d(\mathbf{x}(M), \mathbf{a})>0$ where $d$ is Euclidean distance of $\mathbf{R}^{n}$. Then $M$ has finite total curvature. In addition if $M$ is geodesically complete, then the immersion $\mathbf{x}$ is proper.

The second assertion follows directly from the Jorge-Meeks theorem ([12]). If $M$ is properly immersed, then $M$ is stochastically complete. Moreover if $M$ has quadratic volume growth, $2 Q_{p}(M, \mathbf{a})=V_{p}(M)<\infty$. Hence our result is a generalization of Chen's result.

It is well-known that a properly immersed surface with quadratic volume growth is parabolic (cf. [8]). And it is also true that so is a properly immersed surface with $V_{p}(M)<\infty$. It is the first step of the proof of our result to show $M$ is parabolic under the assumption of the theorem. Thus we first note a relationship between parabolicity and projective volume in section 1 . In section 2 we give a lemma on logarithmic derivative of subharmonic functions which is a main tool for our proof. We give a proof of the theorem there using it. We add some remarks about relationships among stochastic completeness, parabolicity and finiteness of total curvature in section 3 . Especially we note there that the assumption on stochastic completeness can not be removed.

We also remark that the situation in the case that $d(\mathbf{x}(M), \mathbf{a})=0$ is different from the case that $d(\mathbf{x}(M), \mathbf{a})>0$ when $\mathbf{x}$ is improper. To treat the former case we need some amount of discussion. The case is not treated here and will be discussed elsewhere.

We would note that Fujimoto [7] considered an analogue of Nevanlinna theory for minimal surface and obtained a result looking like the second main theorem by Nevanlinna (see also [5]). Though he did not use a lemma on logarithmic derivative, our lemma of logarithmic derivative and our result would be closely related to it as they originally were in Nevanlinna theory for meromorphic functions.

Throughout this note we use some probabilistic notions and technique.

\section{Parabolicity and projective volume}

We say that a Riemannian manifold $M$ is parabolic if it does not have a positive Green function on $M$, or equivalently,

$$
\int_{0}^{\infty} p(t, x, y) d t=\infty, \quad \text { for } x, y \in M,
$$


where $p(t, x, y)$ is the heat kernel for $(1 / 2) \Delta_{M}$. We say that a Riemannian manifold $M$ is stochastically complete if

$$
\int_{M} p(t, x, y) d v(y)=1, \quad \text { for } x \in M,
$$

where $d v$ is the measure defined by Riemannian volume. We remark that parabolic Riemannian manifolds are always stochastically complete. The heat kernel $p(t, x, y)$ defines Brownian motion on $M$.

We first note that

Proposition 2. Let $\mathbf{x}: M \rightarrow \mathbf{R}^{n}$ be a minimal surface. If $M$ is stochastically complete and

$$
Q_{p}(M, \mathbf{a})=\int_{M} \frac{\left|(\mathbf{x}-\mathbf{a})^{\perp}\right|^{2}}{|\mathbf{x}-\mathbf{a}|^{4}} d v<\infty
$$

for some $\mathbf{a} \in \mathbf{R}^{n}$ with $d(\mathbf{x}(M), \mathbf{a})>0$, then $M$ is parabolic.

Let $X$ be a Brownian motion on $M$ starting from $x_{0} \in M$ with respect to the induced metric, $E_{x_{0}}$ denote the expectation with respect to $X$ and $u(x)=$ $\log |\mathbf{x}(x)|$.

By direct calculation and Dynkin's formula (cf. [4]) we have

Lemma 3. Assume $d(\mathbf{x}(M), 0)>0$. We have

and

$$
\frac{1}{2} \Delta_{M} u(x)=\frac{\left|\mathbf{x}^{\perp}\right|^{2}}{|\mathbf{x}|^{4}}
$$

$$
E_{x_{0}}\left[u\left(X_{\tau_{D}}\right)\right]-u\left(x_{0}\right)=E_{x_{0}}\left[\int_{0}^{\tau_{D}} \frac{\left|\mathbf{x}^{\perp}\right|^{2}}{|\mathbf{x}|^{4}}\left(X_{s}\right) d s\right]=\int_{D} g_{D}\left(x_{0}, y\right) \frac{\left|\mathbf{x}^{\perp}\right|^{2}}{|\mathbf{x}|^{4}}(y) d v(y),
$$

where $x_{0} \in D \subset M$ is a relatively compact set, $\tau_{D}=\inf \left\{t>0: X_{t} \notin D\right\}$ and $g_{D}$ is Green function with Dirichlet boundary condition with respect to $(1 / 2) \Delta_{M}$ on $D$.

Proof of Proposition 2. Assume that $\mathbf{a}=0$ for simplicity. Let $\left\{D_{n}\right\}$ be a sequence of sets such that $D_{n} \uparrow M$ and $\bar{D}_{n} \subset D_{n+1}$. Stochastic completeness implies that $\tau_{D_{n}} \rightarrow \infty$ as $n \rightarrow \infty$ a.s.

Assume that $M$ is not parabolic. Then there exists a global Green function $g(x, y)$ on $M$. Finiteness of $Q_{p}(M, 0)$ implies that

$$
\sup _{n} E_{x_{0}}\left[u\left(X_{\tau_{D_{n}}}\right)\right] \leq u\left(x_{0}\right)+\int_{M} g\left(x_{0}, y\right) \frac{\left|\mathbf{x}^{\perp}\right|^{2}}{|\mathbf{x}|^{4}}(y) d v(y)<\infty .
$$

Since $u\left(X_{t}\right)$ is a submartingale bounded from below, by Fatou's lemma

$$
E_{x_{0}}\left[u\left(X_{t}\right)\right]=E_{x_{0}}\left[\liminf _{n \rightarrow \infty} u\left(X_{t \wedge \tau_{D_{n}}}\right)\right] \leq \sup _{n} E_{x_{0}}\left[u\left(X_{\tau_{D_{n}}}\right)\right]<\infty
$$


for $0<t<\infty$. Hence $\sup _{t} E_{x_{0}}\left[u\left(X_{t}\right)\right]<\infty$. By martingale convergence theorem (cf. [14]) $u\left(X_{t}\right)$ converges almost surely to a finite random variable as $t \rightarrow \infty$. On the other hand, from the next proposition we have $\sup _{t} u\left(X_{t}\right)=\infty$, a.s. Thus we arrive at contradiction.

Proposition 4 ([1]). Let $\mathbf{x}: M \rightarrow \mathbf{R}^{n}$ be a stochastic complete minimal surface and $X$ Brownian motion on $M$. Then $\sup _{t}\left|\mathbf{x}\left(X_{t}\right)\right|=\infty$ a.s.

Proof. We first note that $\Delta_{M}|\mathbf{x}(x)|^{2}=4$ since $\mathbf{x}$ is an isometry and a harmonic map. Applying Ito's formula we have

$$
\left|\mathbf{x}\left(X_{t}\right)\right|^{2}-\left|\mathbf{x}\left(X_{0}\right)\right|^{2}=B\left(\left.\left.\int_{0}^{t}|\nabla| \mathbf{x}\right|^{2}\right|^{2}\left(X_{s}\right) d s\right)+2 t,
$$

where $B$ is 1-dimensional standard Brownian motion. On the other hand, $\left.\left.\int_{0}^{t}|\nabla| \mathbf{x}\right|^{2}\right|^{2}\left(X_{s}\right) d s \leq \int_{0}^{t}|\mathbf{x}|^{2}\left(X_{s}\right) d s$ since $|\nabla| \mathbf{x}|| \leq 1$. Law of iterated logarithm of Brownian motion enables us to get the desired conclusion.

\section{Lemma of logarithmic derivative for $\delta$-subharmonic functions on parabolic Riemann surfaces}

If $M$ is a parabolic Riemann surface, there exists an exhaustion function $\rho$ such that $\log \rho$ is harmonic function and the number of critical points of $\rho$ in $\{\rho \leq r\}$ is $-\chi(r)$ for every $r>0$ where $\chi(r)$ is Euler characteristic of $\{\rho \leq r\}$.

We first prove some preliminary lemmas to obtain our lemma of logarithmic derivative. Set $D_{r}=\{\rho<r\}, \partial D_{r}=\{\rho=r\}$ and $D(\varepsilon, r)=\{\varepsilon<\rho<r\}$.

Lemma 5. Let $g_{r}(x, y)$ be Green function on $D_{r}$. For any $x_{0} \in D_{r}$ there exist constants $C_{1}>0, C_{2}>0$ independent of $r$ and $y$ such that

$$
C_{1} \log \frac{r}{\rho(y)} \leq g_{r}\left(x_{o}, y\right) \leq C_{2} \log \frac{r}{\rho(y)} .
$$

Proof. We first see that $\log \rho\left(X_{t}\right) / \rho\left(X_{0}\right)=B\left(\phi_{t}\right)$ where $\phi_{t}=\int_{0}^{t}|\nabla \rho|^{2} / \rho^{2}\left(X_{s}\right) d s$ and $B$ is one dimensional Brownian motion.

Rewriting it as $\rho\left(X_{\phi_{t}^{-1}}\right)=\rho\left(X_{0}\right) e^{2 B(t)}$, we have

$$
\rho\left(X_{t}\right) / \rho\left(X_{0}\right)=r_{\psi_{t}}, \quad \psi_{t}=\int_{0}^{t}|\nabla \rho|^{2}\left(X_{s}\right) d s,
$$

where $r_{t}$ is a 2-dimensional Bessel process.

We can assume that $\rho\left(X_{0}\right)=1$ without loss of generality.

Define $\tau_{r}=\left\{t>0 \mid \rho\left(X_{t}\right) \geq r\right\}$. Take $0<\varepsilon<r \quad$ and $x_{o} \in D_{\varepsilon}$. For $h \in C_{0}((0, \infty))$ 
PARABOLICITY, PROJECTIVE VOLUME AND FINITENESS OF TOTAL CURVATURE

$$
\begin{aligned}
E_{x_{o}}\left[\int_{0}^{\tau_{r}} h\left(\rho\left(X_{t}\right)\right)|\nabla \rho|^{2}\left(X_{t}\right) d t\right] & =E_{x_{o}}\left[\int_{0}^{\tau_{r}} h\left(r_{\psi_{t}}\right) d \psi_{t}\right] \\
& =E_{1}\left[\int_{0}^{\sigma_{r}} h\left(r_{t}\right) d t\right],
\end{aligned}
$$

where $\sigma_{r}=\left\{t>0 \mid r_{t} \geq r\right\}$.

From this we have that there exist $c_{1}>0, c_{2}>0$ such that

$$
c_{1} t \log \frac{r}{t} \leq \int_{\partial D_{t}} g_{r}\left(x_{0}, y\right)|\nabla \rho|(y) d A_{t}(y) \leq c_{2} t \log \frac{r}{t},
$$

holds for $0<t<r$ where $d A_{t}(y)$ is the induced surface measure on $\partial D_{t}$.

Take $\varepsilon \in(0, r)$. By local Harnack inequality on $\partial D_{\varepsilon}$ we can see that there exist $c_{3}>0, c_{4}>0$ independent of $r$ and $y$ such that

$$
c_{3} \log r \leq g_{r}\left(x_{0}, y\right) \leq c_{4} \log r \text { for } y \in \partial D_{\varepsilon} .
$$

Since $g_{r}\left(x_{o}, y\right)$ is a bounded harmonic function on $D(\varepsilon, r)$,

$$
g_{r}\left(x_{o}, y\right)=E_{y}\left[g_{r}\left(x_{o}, X_{\tau_{r} \wedge \tau_{\varepsilon}}\right)\right]=E_{y}\left[g_{r}\left(x_{o}, X_{\tau_{\varepsilon}}\right): \tau_{r}>\tau_{\varepsilon}\right] .
$$

Hence

$$
c_{3}(\log r) P_{y}\left(\tau_{r}>\tau_{\varepsilon}\right) \leq g_{r}\left(x_{o}, y\right) \leq c_{4}(\log r) P_{y}\left(\tau_{r}>\tau_{\varepsilon}\right) .
$$

Combining this with

$$
P_{y}\left(\tau_{r}>\tau_{\varepsilon}\right)=\frac{\log \frac{r}{\rho(y)}}{\log \frac{r}{\varepsilon}}
$$

we have the desired inequality.

LEMMa 6. Let $f$ be a nonnegative locally integrable function on $M$. Then for any $\delta>0$ there exists $E_{\delta} \subset \mathbf{R}$ with $\left|E_{\delta}\right|<\infty$ such that for $r \notin E_{\delta}$,

$$
E_{x_{0}}\left[\frac{f}{|\nabla \rho|^{2}}\left(X_{\tau_{r}}\right)\right] \leq C r^{\delta}\left(E_{x_{0}}\left[\int_{0}^{\tau_{r}} f\left(X_{t}\right) d t\right]\right)^{(1+\delta)^{2}} .
$$

Proof. Set $m_{r}=\int_{\partial D_{r}} f /|\nabla \rho| d A_{r}$ where $d A_{r}$ is the induced surface measure on $\partial D_{r}$. We proceed to carrying out the same calculation as in [2] with the help of Lemma 5. Applying co-area formula we have

$$
\begin{aligned}
E_{x_{0}}\left[\int_{0}^{\tau_{r}} f\left(X_{t}\right) d t\right] & =\int_{D_{r}} g\left(x_{0}, y\right) f(y) d v(y) \\
& =\int_{0}^{r} d t \int_{\partial D_{t}} g\left(x_{0}, y\right) \frac{f}{|\nabla \rho|}(y) d A_{t}(y) .
\end{aligned}
$$


By Lemma 5 we have

$$
C_{1} \int_{0}^{r} \log \frac{r}{t} m_{t} d t \leq E_{x_{0}}\left[\int_{0}^{\tau_{r}} f\left(X_{t}\right) d t\right] \leq C_{2} \int_{0}^{r} \log \frac{r}{t} m_{t} d t .
$$

We first see that Lebesgue measure of the set

$$
E_{\delta}=\left\{r \in(0, \infty) \mid m_{r}>r^{1+\delta}\left(E_{x_{0}}\left[\int_{0}^{\tau_{r}} f\left(X_{t}\right) d t\right]\right)^{(1+\delta)^{2}}\right\}
$$

is finite. Set

$$
E_{\delta}^{(1)}=\left\{r \in(0, \infty) \mid \frac{1}{r} \int_{0}^{r} m_{t} d t>\left(\int_{0}^{r} \log \frac{r}{t} m_{t} d t\right)^{(1+\delta)}\right\} .
$$

Then it is easy to see that $\left|E_{\delta}^{(1)}\right|<\infty$.

$$
\begin{aligned}
\int_{E_{\delta} \cap\left(E_{\delta}^{(1)}\right)^{c} \cap[1, \infty)} d r & \leq C_{1}^{-(1+\delta)^{2}} \int_{\left(E_{\delta}^{(1)}\right)^{c} \cap[1, \infty)} \frac{m_{r}}{r^{\delta+1}\left(\int_{0}^{r} \log \frac{r}{t} m_{t} d t\right)^{(1+\delta)^{2}}} d r \\
& \leq C_{1}^{-(1+\delta)^{2}} \int_{1}^{\infty} \frac{m_{r}}{\left(\int_{0}^{r} m_{t} d t\right)^{1+\delta}} d r<\infty .
\end{aligned}
$$

Thus $\left|E_{\delta}\right|<\infty$.

Harmonic measure $\omega_{x_{0}}$ on $\partial D_{r}$ with respect to $x_{0}$ and $D_{r}$ is obtained by

$$
\int_{\partial D_{r}} f(x) d \omega_{x_{0}}(x)=\int_{\partial D_{r}} f(x) \frac{\partial}{\partial n} g_{r}\left(x_{0}, x\right) d A_{r}(x),
$$

where $\partial / \partial n$ is inward normal derivative on $\partial D_{r}$. Since $n=\nabla \rho /|\nabla \rho|$,

$$
\frac{\partial}{\partial n} \log \frac{r}{\rho(x)}=-\left\langle\nabla \log \frac{r}{\rho(x)}, n\right\rangle=\frac{|\nabla \rho|}{\rho(x)} .
$$

Hence by Lemma 5

$$
C_{1} \int_{\partial D_{r}} \frac{f}{|\nabla \rho|} d A_{r} \leq r \int_{\partial D_{r}} \frac{f}{|\nabla \rho|^{2}} d \omega=r E_{x_{0}}\left[\frac{f}{|\nabla \rho|^{2}}\left(X_{\tau_{r}}\right)\right] \leq C_{2} \int_{\partial D_{r}} \frac{f}{|\nabla \rho|} d A_{r} .
$$

We say $u$ is a $\delta$-subharmonic function if $u$ is a difference of two subharmonic functions locally on $M$. A signed measure $\mu=\mu_{+}-\mu_{-}\left(\mu_{+}, \mu_{-} \geq 0\right)$ uniquely corresponds to a $\delta$-subharmonic function $u$ (cf. [9]). Such a $\mu$ is called a Riesz charge of $u$.

By a little probabilistic argument we have

LEMma 7 ([2]). Let $u$ be a nonnegative $\delta$-subharmonic function on $M$ with Riesz charge $\mu_{+}-\mu_{-}$and $u\left(x_{0}\right)$ be finite. If $\mu_{-}$is not supported by a set of positive capacity, then it holds that 
PARABOLICITY, PROJECTIVE VOLUME AND FINITENESS OF TOTAL CURVATURE 233

$$
E_{x_{0}}\left[\left(\int_{0}^{\tau_{D}}|\nabla u|^{2}\left(X_{S}\right) d s\right)^{\alpha}\right] \leq C_{\alpha}\left(E_{x_{0}}\left[u\left(X_{\tau_{D}}\right)\right]+\int_{D} g_{D}\left(x_{0}, y\right) d \mu_{-}(y)\right)^{2 \alpha}
$$

holds for $0<\alpha<1 / 2$ and any relatively compact domain $D \subset M$.

From these lemmas we have the following theorem in similar way to [2]. Set $u^{+}(x)=\max \{u(x), 0\}$.

THEOREM 8 (Lemma of logarithmic derivative for $\delta$-subharmonic functions.). Let $u$ be a $\delta$-subharmonic function on $M$ and $u\left(x_{0}\right)$ be finite. Suppose that $u^{+}$has Riesz charge $\mu_{+}-\mu_{-}$such that $\mu_{-}$is not supported by a set of positive capacity. Then for any $\delta>0$ there exist constants $C_{1}, C_{2}, C_{3}>0$ and $E_{\delta} \subset \mathbf{R}_{+}$with $\left|E_{\delta}\right|<\infty$ such that

$$
\begin{aligned}
E x_{0}\left[\log ^{+}|\nabla u|\left(X_{\tau_{r}}\right)\right] \leq & C_{1} \log ^{+}\left(E x_{0}\left[u\left(X_{\tau_{r}}\right)\right]+\int_{D} g_{D_{r}}\left(x_{0}, y\right) d \mu_{-}(y)\right) \\
& +C_{2} \log r+C_{3} \int_{1}^{r}-\frac{\chi(t)}{t} d t
\end{aligned}
$$

holds for $r \notin E_{\delta}$.

LEMMA 9. Let $u(x)=\log |\mathbf{x}| . \quad$ If $Q_{p}(M, 0)<\infty$ and $d(\mathbf{x}(M), 0)>0$, then

$$
E_{x_{0}}\left[u\left(X_{\tau_{r}}\right)\right]-u\left(x_{0}\right)=E_{x_{0}}\left[\int_{0}^{\tau_{r}} \frac{\left|\mathbf{x}^{\perp}\right|^{2}}{|\mathbf{x}|^{4}}\left(X_{s}\right) d s\right]=O(\log r) .
$$

Proof. Apply co-area formula and note the form of Green function on $D_{r}$.

Proof of Theorem 1. Let $\mathbf{x}: M \rightarrow \mathbf{R}^{3}$ be a minimal surface. Assume that $\mathbf{a}=0$ for simplicity. Set $u(x)=\log |\mathbf{x}|$. We first note that

$$
|\nabla u|^{2}=\frac{\left|\mathbf{x}^{\top}\right|^{2}}{|\mathbf{x}|^{2}}
$$

where $\mathbf{x}^{\top}$ is a projection of $\mathbf{x}$ on the tangential space to the surface $\mathbf{x}(M)$ at a point $\mathbf{x}(x)$. Note that $|\mathbf{x}|^{2}=\left|\mathbf{x}^{\top}\right|^{2}+\left|\mathbf{x}^{\perp}\right|^{2}$.

We apply Theorem 8 and Lemma 3 to this $u$. Then we have

$$
E_{x_{0}}\left[\log \frac{\left|\mathbf{x}^{\top}\right|}{|\mathbf{x}|^{2}}\left(X_{\tau_{r}}\right)\right] \leq \text { const. } \log ^{+} E_{x_{0}}\left[\int_{0}^{\tau_{r}} \frac{\left|\mathbf{x}^{\perp}\right|^{2}}{|\mathbf{x}|^{4}}\left(X_{s}\right) d s\right]+O(\log r),
$$

for $r$ outside the exceptional set. On the other hand, we proceed to making direct calculation and similar way to Lemma 9 to see that

$$
E_{x_{0}}\left[\log \left|\mathbf{x}^{\top}\right|^{2}\left(X_{\tau_{r}}\right)\right]=\text { const. } \int_{\delta}^{r} \frac{K(t)}{t}+\text { const }
$$


for $0<\delta<r$ where $K(t)=\int_{\rho \leq t}-k(x) d v$ with the Gaussian curvature $k(x)$. Hence with Lemma 9 we have

$$
\int_{\delta}^{r} \frac{K(t)}{t}=O(\log r)
$$

which implies finiteness of total curvature.

\section{Remarks}

We give some remarks on the assumptions of Theorem 1 .

1. We first point out that if $M$ is geodesically complete and of finite total curvature, then $M$ is parabolic. This well-known fact is originally due to Huber. We can see this from Picard type theorem of Gauss map due to Fujimoto ([7], Theorem $6.10, \mathrm{p} 124$.). Let us assume that $M$ is not parabolic and the total curvature of $M$ is finite. Then the characteristic function in the sense of Nevanlinna of Gauss map $G$ of $M$ is bounded. This implies $G$ omits a set of hyperplanes of positive capacity. This contradicts completeness of $M$ by $\mathrm{Fu}-$ jimoto's theorem.

2. There exists a minimal surface which is geodesically incomplete, of finite total curvature and not parabolic. We can construct such surfaces by Weierstrass formula. We choose a meromorphic function $g$ on the unit disk in $\mathbf{C}$ such that its image area is finite. We take it as a Gauss map. By Weierstrass formula there exists a minimal surface in $\mathbf{R}^{3}$ which Gauss map is $g$. It has finite total curvature and is not parabolic and geodesically incomplete by a similar argument to 1 of this remark.

3. We also note there exist minimal surfaces which are geodesically complete and stochastically incomplete. It is known that there exist bounded and geodesically complete minimal surfaces immersed in $\mathbf{R}^{n}$. As for the construction of such minimal surfaces, see [11] for the case $n=6,8$ and [13] for $n=3$. Both of them considered immersions from a unit disc in $\mathbf{C}$.

We have that such surfaces are stochastically incomplete and not parabolic and of infinite total curvature. As we saw, boundedness of minimal surfaces implies stochastic incompleteness. In particular it is not parabolic. The second assertion follows from 1 in this remark.

4. We note a little about $Q_{p}$. We consider a hypersurface $M=\left\{(z, w) \in \mathbf{C}^{2} \mid g(z)=w\right\}$ in $\mathbf{C}^{2}$ where $g$ is a holomorphic function on $D$ : unit disk in C. Then $D$ has the metric $d \sigma^{2}=\left(\left|g^{\prime}(z)\right|^{2}+1\right)|d z|^{2}$ induced by $\mathbf{x}: D \rightarrow M \subset \mathbf{C}^{2}$. We can see

Hence

$$
\left|\mathbf{x}^{\perp}\right|^{2}=\frac{2\left|z g^{\prime}(z)+w\right|^{2}}{\sqrt{\left|g^{\prime}(z)\right|^{2}+1}} .
$$




$$
\begin{aligned}
Q_{p}(M, 0) & =\int_{D} \frac{\left|\mathbf{x}^{\perp}\right|^{2}}{|\mathbf{x}|^{4}} d \sigma^{2} \\
& \leq \int_{D} \frac{\left(\left|g^{\prime}(z)\right|^{2}+|g(z)|^{2}\right)}{\left(|z|^{2}+|g(z)|^{2}\right)^{2}}|d z|^{2} .
\end{aligned}
$$

It follows from this that for such a hypersurface $M, Q_{p}(M, 0)<\infty$ if and only if $\int_{M} g^{*} \omega_{\mathbf{P}^{1}}<\infty$ where $\omega_{\mathbf{P}^{1}}$ is Fubini-Study metric on $\mathbf{P}^{1}$ : one dimensional complex projective space. It is easy to see that this surface is not stochastically complete since $\lim _{t \rightarrow \zeta} g\left(X_{t}\right)$ exists. There are a lot of holomorphic functions on $D$ satisfying this condition. It would be a problem whether there is a geodesically complete surface among these hypersurfaces or not.

5. We can construct a minimal surface which is geodesically incomplete and stochastically complete using Weierstrass formula. This surface has Gauss map with bounded image. The details will be in [3].

6. Our last remark is about properness and stochastic completeness. As we mentioned, properness implies stochastic completeness and geodesic completeness. Then a natural question may arise, asking if there exists an improper, stochastically and geodesically complete minimal surface.

Proposition 10. There exists a geodesically and stochastically complete and improper minimal surface in $\mathbf{R}^{3}$.

We already gave a non-flat, geodesically and stochastic complete minimal surface between two parallel planes ([1]). By the half-space theorem due to Hoffman and Meeks ([10]) this must be improper.

There are a lot of minimal surfaces which are not only stochastically but also geodesically complete.

\section{REFERENCES}

[1] Atsuji, Atsushi, Remarks on harmonic maps into a cone from a stochastically complete manifold, Proc. Japan Acad. Ser. A Math. Sci. 75 (1999), no. 7, 105-108.

[2] Atsuji, Atsushi, A lemma of logarithmic derivative for some $\delta$-subharmonic functions, Complex Variables Theory Appl. 46 (2001), no. 3, 195-206.

[3] Atsuji, Atsushi, On stochastically complete minimal submanifolds, in preparation.

[4] Bass, Richard F., Probabilistic techniques in analysis. Probability and its Applications, Springer-Verlag, New York, 1995.

[5] Beckenbach, E. F. and Cootz, T. A., The second fundamental theorem for meromorphic minimal surfaces, Bull. Amer. Math. Soc. 76 (1970), 711-716.

[6] Chen, Qing, On the total curvature and area growth of minimal surfaces in $\mathbf{R}^{n}$, Manuscripta Math. 92 (1997), no. 2, 135-142.

[ 7 ] Fujimoto, Hirotaka, Nevanlinna theory and minimal surfaces, Geometry, V, 95-151, 267272, Encyclopedia Math. Sci., 90, Springer, Berlin, 1997.

[ 8 ] Grigor'yan, Alexander, Analytic and geometric background of recurrence and non-explosion of the Brownian motion on Riemannian manifolds, Bull. Amer. Math. Soc. (N.S.) 36 (1999), no. 2, 135-249. 
[ 9 ] Hayman, W. K., Subharmonic functions. Vol. 2, London Mathematical Society Monographs, 20. Academic Press, London, 1989.

[10] Hoffman, D. and Meeks, William H., III, The strong halfspace theorem for minimal surfaces, Invent. Math. 101 (1990), 373-377.

[11] Jones, Peter W., A complete bounded complex submanifold of $C^{3}$, Proc. Amer. Math. Soc. 76 (1979), no. 2, 305-306.

[12] Jorge, Luquesio P. And Meeks, William H., III, The topology of complete minimal surfaces of finite total Gaussian curvature, Topology 22 (1983), no. 2, 203-221.

[13] Nadirashvili, Nikolai, Hadamard's and Calabi-Yau's conjectures on negatively curved and minimal surfaces, Invent. Math. 126 (1996), no. 3, 457-465.

[14] Revuz, Daniel and Yor, Marc, Continuous martingales and Brownian motion, Third edition. Grundlehren der Mathematischen Wissenschaften, 293. Springer-Verlag, Berlin, 1999.

[15] Tkachev, Vladimir G., Finiteness of the number of ends of minimal submanifolds in Euclidean space, Manuscripta Math. 82 (1994), no. 3-4, 313-330.

\section{KeIO UNIVERSITY}

4-1-1, Hiyoshi

YOKOHAMA, 223-8521

JAPAN

E-mail address: atsuji@math.hc.keio.ac.jp 